\title{
ON CERTAIN MAPPINGS OF RIEMANNIAN MANIFOLDS
}

\author{
MINORU KURITA
}

In this paper we consider certain tensors associated with differentiable mappings of Riemannian manifolds and apply the results to a $p$-mapping, which is a special case of a subprojective one in affinely connected manifolds (cf. [1], [7]). The $p$-mapping in Riemannian manifolds is a generalization of a conformal mapping and a projective one. From a point of view of differential geometry an analogy between these mappings is well known. On the other hand it is interesting that a stereographic projection of a sphere onto a plane is conformal, while a central projection is projectve, namely geodesic-preserving. This situation was clarified partly in [6]. A $p$-mapping defined in this paper gives a precise explanation of this and also affords a certain mapping in the euclidean space which includes a similar mapping and an inversion as special cases.

\section{Tensors associated with mappings of Riemannian manifolds}

1. Let $M$ and $N$ be two $n$-dimensional Riemannian manifolds of differentiable class $C^{3}$ and $\varphi$ be a mapping of differentiable class $C^{3}$ which is locally regular. We take a coordinate neighborhood $U$ and differentiable sets of orthogonal coframes on $U$ and $\varphi(U)$. Then the arc-elements of $M$ and $N$ are given respectively as

$$
d s^{2}=\sigma^{i} \sigma^{i}, \quad d t^{2}=\tau^{i} \tau^{i}
$$

Now we put

$$
\varphi^{*} \tau^{i}=p_{j}^{i} \sigma^{j}, \quad a_{i j}=p_{i}^{k} p_{j}^{k} .
$$

Then we have $\operatorname{det}\left(a_{i j}\right) \neq 0$ on account of regularity and

$$
\varphi^{*} d t^{2}=\varphi^{*}\left(\tau^{i} \tau^{i}\right)=a_{i j} \sigma^{i} \sigma^{j}, \quad\left(a_{i j}=a_{j i}\right) .
$$

We call $A=\left(a_{i j}\right)$ the first tensor of our mapping $\varphi$.

Next we take forms of Riemannian connections

Received February 21, 1964. 


$$
\sigma=\left(\sigma_{j}^{i}\right), \quad \tau=\left(\tau_{j}^{i}\right)
$$

of $M$ and $N$ which are defined by the relations

$$
d \sigma^{i}=\sigma^{j} \wedge \sigma_{j}^{i} \quad\left(\sigma_{j}^{i}=-\sigma_{i}^{j}\right), \quad d \tau^{i}=\tau^{j} \wedge \tau_{j}^{i} \quad\left(\tau_{j}^{i}=-\tau_{i}^{j}\right) .
$$

Throughout this paper we assume that for matrices such as $\sigma=\left(\sigma_{j}^{i}\right), \tau=\left(\tau_{j}^{i}\right)$ an upper index denotes a number of column and a lower one a number of row.

$\varphi^{*} d t^{2}$ can be considered as a new Riemannian metric on $M$. It is represented as (1.3) and when we put $P=\left(p_{j}^{i}\right)$, the form $\left.\lambda=\lambda_{j}^{i}\right)$ of the Riemannian connection with respect to the base $\sigma^{i}$ is given by

$$
\lambda=P \cdot \varphi^{*} \tau \cdot P^{-1}+d P P^{-1}
$$

and satisfies the relation

$$
d \sigma^{i}=\sigma^{j} \wedge \lambda_{j}^{i}
$$

We have by (1.5) and (1.7) $\sigma^{j} \wedge\left(\lambda_{j}^{i}-\sigma_{j}^{i}\right)=0$ and when we put

$$
\gamma=\left(\gamma_{j}^{i}\right)=\lambda-\sigma, \quad \gamma_{j}^{i}=c_{j k}^{i} \sigma^{k}
$$

we get

$$
\sigma^{j} \wedge \gamma_{j}^{i}=0, \text { namely } c_{j k}^{i}=c_{k j}^{i}
$$

As $\sigma$ and $\lambda$ are forms of two Riemannian connections with respect to the same base $\sigma^{1}, \ldots, \sigma^{n}$, their difference $\gamma=\left(\gamma_{j}^{i}\right)=\left(c_{j k}^{i} \sigma^{k}\right)$ is a tensorial form on $M$, which we call the second tensor of our mapping. It plays the most important role in our theory.

Next we put the curvature forms on $M$ and $N$ as

$$
\Sigma=\left(\sum_{j}^{i}\right)=d \sigma-\sigma \wedge \sigma, \quad T=\left(T_{j}^{i}\right)=d \tau-\tau \wedge \tau .
$$

We have by (1.6) and (1.8)

$$
r=P \cdot \varphi^{*} \tau \cdot P^{-1}+d P P^{-1}-\sigma,
$$

namely

$$
d P=(\gamma+\sigma) P-P \cdot \varphi^{*} \tau .
$$

Taking the outer differential we get

$$
0=(d \gamma+d \sigma) P-(\gamma+\sigma) \wedge d P-d P \wedge \varphi^{*} \tau-P \varphi^{*}(d \tau) .
$$

Substituting (1.12) we obtain by (1.10)

$$
\Gamma=P \cdot \varphi^{*} T \cdot P^{-1}-\Sigma
$$


where we have put

$$
\Gamma=\left(\Gamma_{j}^{i}\right)=d \gamma-\gamma \wedge \sigma-\sigma \wedge \gamma-\gamma \wedge \gamma .
$$

We call this tensor $\Gamma$ the third tensor of our mapping.

$$
\begin{aligned}
& \text { Putting } \quad \sum_{j}^{i}=\frac{1}{2} S_{j k h}^{i} \sigma^{k} \wedge \sigma^{h}, \quad T_{j}^{i}=\frac{1}{2} T_{j k h}^{i} \tau^{k} \wedge \tau^{h}, \quad \Gamma_{j}^{i}=\frac{1}{2} C_{j k h}^{i} \sigma^{k} \wedge \sigma^{h} \\
& \left(S_{j k h}^{i}=-S_{j h k}^{i}, \quad T_{j k h}^{i}=-T_{j h k}^{i}, \quad C_{j k h}^{i}=-C_{j h k}^{i}\right)
\end{aligned}
$$

and

$$
\left(p_{j}^{i}\right)^{-1}=\left(P_{j}^{i}\right)
$$

we get

$$
C_{j k h}^{i}=p_{j}^{l} p_{k}^{m} p_{h}^{n} P_{u}^{i} \cdot \varphi^{*} T_{l m n}^{u}-S_{j k h}^{i}
$$

and

$$
C_{j k h}^{i}=\nabla_{k} c_{j h}^{i}-\nabla_{h} c_{j k}^{i}-c_{j k}^{l} c_{l h}^{i}+c_{j h}^{l} c_{l k}^{i}
$$

where $\nabla$ means a covariant differentiation with respect to the Riemannian connection $\sigma=\left(\sigma_{j}^{i}\right)$.

2. Now we calculate $c_{j k}^{i}$. As $\lambda=\left(\lambda_{j}^{i}\right)$ are forms of Riemannian connection of $\varphi^{*} d t^{2}=a_{i j} \sigma^{i} \sigma^{j}$, the tensor $A=\left(a_{i j}\right)$ is parallel with respect to the connection. Hence we have

$$
d a_{i j}=a_{k j} \lambda_{i}^{k}+a_{i k} \lambda_{j}^{k}
$$

As $\lambda_{j}^{i}=\gamma_{j}^{i}+\sigma_{j}^{i}$ by (1.8), we get

$$
\begin{aligned}
D a_{i j} & =d a_{i j}-a_{k j} \sigma_{i}^{k}-a_{i k} \sigma_{j}^{k}=a_{k j} \gamma_{i}^{k}+a_{i k} \gamma_{j}^{k} \\
& =a_{k j} c_{i h}^{k} \sigma^{h}+a_{i k} c_{j h}^{k} \sigma^{h},
\end{aligned}
$$

where $D a_{i j}$ is a covariant differential of $a_{i j}$. Hence, putting

$$
D a_{i j}=\nabla_{k} a_{i j} \sigma^{k}=a_{i j k} \sigma^{k}
$$

we get

$$
a_{i j h}=a_{k j} c_{i h}^{k}+a_{i k} c_{j h}^{k}
$$

If we put $A_{i h j}=a_{k j} c_{i h}^{k}$, we have $a_{i j h}=A_{i h j}+A_{j h i}$, and by virtue of (1.9) $A_{i j h}$ $=A_{j i h} . \quad$ Hence

$$
a_{i j h}+a_{h j i}-a_{h i j}=2 A_{i h j}=2 a_{k j} c_{i h}^{k} .
$$

So, by putting

$$
A^{-1}=\left(a_{i j}\right)^{-1}=\left(a^{i j}\right)
$$

we obtain 


$$
c_{i h}^{k}=\frac{1}{2} a^{k j}\left(a_{i j h}+a_{h j i}-a_{h i j}\right)
$$

Thus we can calculate $c_{j k}^{i}$ from $a_{i j}$.

We contract (1.19) and denote the resulting vector by $c_{i}$. Then

$$
c_{i}=c_{i h}^{h}=\frac{1}{2} a^{h j}\left(a_{i j h}+a_{h j i}-a_{h i j}\right)=\frac{1}{2} a^{h j} a_{h j i}
$$

by virtue of $a_{i j h}=a_{j i h}, a^{h j}=a^{j h}$. Hence

$$
\begin{aligned}
c_{i} \sigma^{i} & =\frac{1}{2} a^{h j} a_{h j i} \sigma^{i}=\frac{1}{2} a^{h j} D a_{h j} \\
& =\frac{1}{2} a^{h j}\left(d a_{h j}-a_{k j} \sigma_{h}^{k}-a_{h k} \sigma_{j}^{k}\right)=\frac{1}{2} a^{h j} d a_{h j} .
\end{aligned}
$$

When we put

$$
\Delta=\operatorname{det}\left(a_{i j}\right)
$$

we have $d \Delta=a^{h j} \Delta \cdot d a_{h j}$, and hence

$$
c_{i} \sigma^{i}=\frac{1}{2} \Delta^{-1} d \Delta, \quad \text { namely } \quad c_{i}=\nabla_{i}(\log \sqrt{\Delta}) .
$$

As $\sigma^{i}$ is an orthogonal base, $s^{i}=c_{h h}^{i}$ are components of a vector. By (1.19) we get

$$
s^{i}=c_{h h}^{i}=a^{i j}\left(a_{h j h}-\frac{1}{2} a_{h h j}\right)
$$

We contract (1.15) with respect to $i$ and $h$ and put

$$
S_{j k}=S_{j k i}^{i}, \quad T_{j k}=T_{j k i}^{i}, \quad C_{j k}=C_{j k i}^{i} .
$$

Then we get

$$
C_{j k}=p_{j}^{l} p_{k}^{m} \cdot \varphi^{*} T_{l m}-S_{j k}
$$

and

$$
C_{j k}=\nabla_{k} c_{j}-\nabla_{h} c_{j k}^{h}-c_{j k}^{l} c_{l}+c_{j h}^{l} c_{l k}^{h} .
$$

Contracting (1.23) with respect to $j, k$ and putting $S=S_{i i}$ we get

$$
\nabla_{h} c_{h}-\nabla_{h} s^{h}-s^{l} c_{l}+c_{i h}^{l} c_{l i}^{h}=p_{i}^{l} p_{i}^{m} \cdot \varphi^{*} T_{l m}-S .
$$

These formulas except (1.21) are not utilised in this paper, but may be useful for further investigations. In fact, in special casses of conformal mapping and projective mapping, they are frequently used.

3. We assume that in $A=\left(a_{i j}\right) i$ is a number of row and $j$ is that of 
column. Then (1.17) can be written as

$$
d A=\lambda A+A^{t} \lambda .
$$

By taking an outer differential we get

$$
0=d \lambda \cdot A-\lambda \wedge d A+d A \wedge^{t} \lambda+A \cdot d^{t} \lambda,
$$

and by substituting (1.26) we get

$$
(d \lambda-\lambda \wedge \lambda) A+A^{t}(d \lambda-\lambda \wedge \lambda)=0 .
$$

As we have $\lambda=\gamma+\sigma$ by (1.8), we get by (1.10) and (1.14)

$$
d \lambda-\lambda \wedge \lambda=d(\gamma+\sigma)-(\gamma+\sigma) \wedge(\gamma+\sigma)=\Gamma+\sum \text {. }
$$

Hence

$$
\left(\Gamma+\sum\right) A+A^{t}\left(\Gamma+\sum\right)=0 .
$$

This is an integrability condition of (1.26) for $a_{i j}$.

4. Here we consider three tensors of a mapping

$$
A=\left(a_{i j}\right), \quad \gamma=\left(\gamma_{j}^{i}\right)=\left(c_{j k}^{i} \sigma^{k}\right), \quad \Gamma=\left(\Gamma_{j}^{i}\right)=\left(\frac{1}{2} C_{j k h}^{i} \sigma^{k} \wedge \sigma^{h}\right)
$$

in some special cases.

(1) isometry

This is characterised by $A=\left(\delta_{i j}\right)$. We have in this case $\gamma=0$ and $\Gamma=0$ by (1.19) and (1.14).

(2) volume-preserving mapping

This is characterised by $\Delta=\operatorname{det} A=1$. In this case we have $c^{i}=0$.

(3) conformal mapping

This is characterised by $A=\left(a \delta_{i j}\right)$. We have in this case $D a_{i j}=d a \cdot \hat{o}_{i j}$, and putting

$$
l=\log \sqrt{a}, \quad d l=l_{i} \sigma^{i}
$$

we get $a_{i j k}=2 l_{k} a \delta_{i j}$. Hence by (1.17)

$$
\begin{aligned}
& c_{j k}^{i}=\delta_{j}^{i} l_{k}+\delta_{k}^{i} l_{j}-\delta_{j}^{k} l_{i} \\
& \gamma_{j}^{i}=c_{j k}^{i} \sigma^{k}=\delta_{j}^{i} d l+l_{j} \sigma^{i}-l_{i} \sigma^{k} .
\end{aligned}
$$

By (1.28) we get in our case

$$
c_{i}=n l_{i}, \quad s^{i}=-(n-2) l_{i} .
$$

Conversely we can not deduce from (1.29) that our mapping is conformal. 
This we will take up later.

(4) affine mapping

This is a mapping which preserves an affine property of our Riemannian connection and as we see by (1.11) it is characterised by

$$
\gamma=0 .
$$

This mapping has been treated by several authors (cf. [3], [4], [8]). If $M$ is a direct product of irreducible Riemannian manifolds $M_{k}$, our mapping is homothetic on each components $M_{k}$.

(5) projective mapping

This is a mapping which preserves geodesics and it it well known that the mapping is characterised by

$$
c_{j k}^{i}=\delta_{j}^{i} l_{k}+\delta_{k}^{i} l_{j} \quad\left(d l=l_{i} \sigma^{i}\right),
$$

namely

$$
\gamma_{j}^{i}=\delta_{j}^{i} d l+l_{j} \sigma^{i}
$$

In this case

$$
c_{i}=(n+1) l_{i}, \quad s^{i}=2 l_{i},
$$

(6) mapping with $Y=0$

In this case we have by (1.13)

$$
\Sigma=P \cdot \varphi^{*} T \cdot P^{-1}
$$

We construct from $\sum=\left(\sum_{j}^{i}\right)$ forms such that

$$
\begin{aligned}
& \mathrm{S}_{4}=\sum_{j}^{i} \wedge \sum_{i}^{j}, \quad S_{8}=\sum_{j}^{i} \wedge \sum_{k}^{j} \wedge \sum_{h}^{k} \wedge \sum_{i}^{h}, \ldots \\
& \quad S_{0}=\varepsilon_{i_{1} \ldots i_{n}} \sum_{i_{2}}^{i_{1}} \wedge \sum_{i_{4}}^{i_{3}} \wedge \cdots \wedge \sum_{i_{n}}^{i_{n-1}} .
\end{aligned}
$$

These are known to be closed forms on $M$ and especially $S_{0}$ is a form which is fundamental in the theory of generalised Gauss-Bonnet's theorem by S. S. Chern (cf. [2]). When we construct corresponding forms $T_{4}, T_{8}, \ldots, T_{0}$ on $N$, we get by (1.32)

$$
S_{4}=\varphi^{*} T_{4}, \quad S_{8}=\varphi^{*} T_{8}, \ldots, \quad S_{0}=\varphi^{*} T_{0} .
$$

In the case when $M$ is of dimension 2 we have $S_{0}=2 K d A$ only, where $K$ denotes the Gaussian curvature and $d A$ an areal element on $M$, and our mapping is a one preserving $K d A$.

If $M$ and $N$ are flat Riemannian manifolds, we have always $\Gamma=0$ by virtue 
of (1.13). But in this case (1.33) means nothing because $S_{4}, S_{3}, \ldots, S_{0}, T_{4}$, $T_{8}, \ldots, T_{0}$ all vanish.

5. Let $L, M, N$ be three Riemannian manifolds of dimension $n$ and $\psi, \varphi$ be mappings $\psi: L \rightarrow M, . \varphi: M \rightarrow N$. We denote the arc-elements of $L, M, N$ by $d r^{2}=\rho^{i} \rho^{i}, d s^{2}=\sigma^{i} \sigma^{i}, d t^{2}=\tau^{i} \tau^{i}$ and put

$$
\psi^{*} \sigma^{i}=g_{j}^{i} \rho^{j}, \quad Q=\left(q_{j}^{i}\right), \quad \varphi^{*} \tau^{i}=p_{j}^{i} \sigma^{j}, \quad P=\left(p_{j}^{i}\right) .
$$

We denote connection forms of $d r^{2}, d s^{2}, d t^{2}$ by $\rho=\left(\rho_{j}^{i}\right), \sigma=\left(\sigma_{j}^{i}\right), \tau=\left(\tau_{j}^{i}\right)$ with respect to the base $\rho^{i}, \sigma^{i}, \tau^{i}$. Then the second tensors $\beta=\left(\beta_{j}^{i}\right)$ of the mapping $\psi$ and $\gamma=\left(\gamma_{j}^{i}\right)$ of $\varphi$ are given by

$$
\beta=Q \sigma Q^{-1}+d Q Q^{-1}-\rho, \quad \gamma=P \tau P^{-1}+d P P^{-1}-\sigma .
$$

We drop here $\phi^{*}, \rho^{*}$ for convenience. Eliminating $\sigma$ from (1.35) we get

$$
\begin{aligned}
\beta & =Q\left(P \tau P^{-1}+d P P^{-1}-\gamma\right) Q^{-1}+d Q Q^{-1}-\rho \\
& =(Q P) \tau(Q P)^{-1}+d(Q P)(Q P)^{-1}-Q \gamma Q^{-1}-\rho
\end{aligned}
$$

and so

$$
\beta+Q_{\gamma} Q^{-1}=(Q P)_{\tau}(Q P)^{-1}+d(Q P)(Q P)^{-1}-\rho .
$$

This gives the second tensor of the mapping $\varphi \circ \psi: L \rightarrow N$. (1.36) is fundamental for a superposition of mappings.

We will answer here the question raised in 4 (3). Let $\varphi: M \rightarrow N$ be a mapping such that

$$
\gamma_{j}^{i}=\delta_{j}^{i} d l+l_{j} \sigma^{i}-l_{i} \sigma^{j} \quad\left(d l=l_{i} \sigma^{i}\right) .
$$

We take a manifold $L$ diffeomorphic to $M$ and consider a conformal diffeomorphic mapping $\psi^{-1}: M \rightarrow L$ with the first tensor $\left(a^{\frac{1}{2}} \delta_{i j}\right)=\left(e^{l} \delta_{i j}\right)$. We take base $\rho^{i}$ in $L$ in such a way that $\rho^{i}=a^{-\frac{1}{2}} \sigma^{i}$. Then for a mapping $\phi: L \rightarrow M$ we have the first tensor $a^{-1} \delta_{i j}=e^{-2 l} \delta_{i j}$ and so

$$
d\left(\log a^{-\frac{1}{2}}\right)=-d l=-l_{i} \sigma^{i}=-a^{-\frac{1}{2}} l_{i} \rho^{i},
$$

and the second tensor is given by

$$
\beta_{j}^{i}=-\delta_{j}^{i} d l-a^{-\frac{1}{2}} l_{j} \rho^{i}+a^{-\frac{1}{2}} l_{i} \rho^{j}=-\delta_{j}^{i} d l-l_{j} \sigma^{i}+l_{i} \sigma^{j} .
$$

Here we have $Q=\left(a^{-1} \delta_{i j}\right)$ and the second tensor of $\varphi \circ \psi$ is by (1.36), (1.37), (1.38) 


$$
\beta+Q \gamma Q^{-1}=\beta+\gamma=0 .
$$

Hence the mapping $f=\varphi \circ \psi$ is affine. Thus we get $\varphi=(\varphi \circ \psi) \circ \psi^{-1}=f \circ \psi^{-1}$ and the following is proved.

TheOREM 1. A mapping $\varphi$ whose second tensor $\gamma=\left(\gamma_{j}^{i}\right)$ is of the form (1.37) is $\varphi=f \circ \psi^{-1}$ with $\psi^{-1}$ conformal and $f$ affine.

\section{Properties of pseudo-projective mappings}

6. In this section we consider a mapping $\varphi: M \rightarrow N$ whose second tensor has the form

$$
\gamma_{j}^{i}=\delta_{j}^{i} d l+l_{j} \sigma^{i}-m_{i} \sigma^{j} \quad\left(d l=l_{i} \sigma^{i}\right) .
$$

As we see from (1.29), (1.31) a conformal mapping and a projective mapping are special cases of our mapping. Other examples will be given in section 3 . From (2.1) we have

$$
c_{j k}^{i}=\delta_{j}^{i} l_{k}+\delta_{k}^{i} l_{j}-\delta_{k}^{j} m_{i}
$$

Contracting with respect to $i, k$ and $j, k$ we get

$$
c_{j}=(n+1) l_{j}-m_{j}, \quad s^{i}=2 l_{i}-n m_{i} .
$$

$\sqrt{\Delta}$ defined by $(1.20)$ is a magnification factor of the volume elements of $M$ and $N$, and is a function globally defined on $M$. Hence by (1.20) $\left(c_{i}\right)$ is a gradient vector defined globally. By virtue of (2.3) we get the following results.

THEOREM 2. $\left(m_{i}\right)$ in (2.1) is a gradient vector, and if $\left(l_{i}\right)$ is a gradient vector of a function globally defined, then so is true for $\left(m_{i}\right)$. In the case of projective mapping $\left(l_{i}\right)$ is a gradient vector of a function globally defined on $M$.

The last assertion is evident by $(2.3)$ because here $m_{j}=0$.

Next we calculate $T=\left(\Gamma_{j}^{i}\right)$. We substitute (2.1) into

$$
\Gamma_{j}^{i}=d \gamma_{j}^{i}-\gamma_{j}^{k} \wedge \sigma_{k}^{i}-\sigma_{j}^{k} \wedge \gamma_{k}^{i}-\gamma_{j}^{k} \wedge \gamma_{k}^{i}
$$

given by (1.14). Putting

$$
D l_{i}=d l_{i}-l_{i} d l, \quad D m_{i}=d m_{i}-m_{i} i m, \quad d m=m_{i} \sigma^{i}
$$

and taking the relations $d \sigma^{i}=\sigma^{j} \wedge \sigma_{j}^{i}, \sigma_{j}^{i}=-\sigma_{i}^{j}$ into consideration we get

$$
\Gamma_{j}^{i}=\left(D l_{j}-l_{j} d l\right) \wedge \sigma^{i}-\left(D m_{i}-m_{i} d m\right) \wedge \sigma^{j}+l_{k} m_{k} \sigma^{j} \wedge \sigma^{i}
$$


Hence we get

$$
\begin{aligned}
& C_{j k h}^{i}=\left(\nabla_{k} l_{j}-l_{j} l_{k}\right) \delta_{h}^{i}-\left(\nabla_{h} l_{j}-l_{j} l_{h}\right) \delta_{k}^{i} \\
& \quad-\left(\nabla_{k} m_{i}-m_{i} m_{k}\right) \delta_{h}^{j}+\left(\nabla_{h} m_{i}-m_{i} m_{h}\right) \delta_{k}^{j}+l_{p} m_{p}\left(\delta_{k}^{j} \delta_{h}^{i}-\delta_{h}^{j} \delta_{k}^{i}\right) .
\end{aligned}
$$

Contracting with respect to $i$ and $h$ we get

$$
\begin{aligned}
C_{j k}=(n-1)\left(\nabla_{k} l_{j}-l_{j} l_{k}\right)-\left(\nabla_{k} m_{j}-m_{j} m_{k}\right) \\
+\left(\nabla_{h} m_{h}-m_{h} m_{h}+(n-1) l_{p} m_{p}\right) \delta_{k}^{j} .
\end{aligned}
$$

Again contracting with respect to $j$ and $k$

$$
C=C_{j j}=(n-1)\left(\nabla_{i} l_{i}-l_{i} l_{i}+\nabla_{i} m_{i}-m_{i} m_{i}+n l_{i} m_{i}\right) .
$$

7. Here we will give a geometric meaning of (2.1). We take a curve $c$ on $M$ with a parameter $u$ and consider the image $\varphi(c)$ of $c$ under our mapping which satisfies the relation (2.1). As was defined in $1\left(\lambda_{j}^{i}\right)$ are forms of Riemannian connection of the Riemannian metric $\varphi^{*} d t^{2}=a_{i j} \sigma^{i} \sigma^{j}$ introduced on $M . \quad \psi=$ $\varphi^{-1}$ can locally be defined and $\left(\psi^{*} \lambda_{j}^{i}\right)$ are connection forms of the Riemannian connetion of the metric $d t^{2}$ on $N$ with respect to the base $\psi^{*} \sigma^{i}$. We drop hereafter $\psi^{*}$ for convenience. By virtue of (1.8) and (2.1) we have

$$
\lambda_{j}^{i}=\sigma_{j}^{i}+\delta_{j}^{i} d l+l_{j} \sigma^{i}-m_{i} \sigma^{j} .
$$

Along the curve $c$ we have

$$
\frac{d}{d u}\left(\frac{\sigma^{i}}{d u}\right)+\frac{\sigma^{j}}{d u} \frac{\lambda_{j}^{i}}{d u}=\frac{d}{d u}\left(\frac{\sigma^{i}}{d u}\right)+\frac{\sigma^{j}}{d u} \frac{\sigma_{j}^{i}}{d u}+2 \frac{d l}{d u} \frac{\sigma^{i}}{d u}-m_{i}\left(\frac{d s}{d u}\right)^{2} .
$$

Denoting the covariant differential by $D_{M}$ and $D_{N}$ respectively according to the metric $d s^{2}$ of $M$ and $d t^{2}$ of $N$ we get

$$
\frac{D_{N}}{d u}\left(\frac{\sigma^{i}}{d u}\right)=\frac{D_{u}}{d u}\left(\frac{\sigma^{i}}{d u}\right)-m_{i}\left(\frac{d s}{d u}\right)^{2}+2 \frac{d l}{d u} \frac{\sigma^{i}}{d u} .
$$

Now we take a curve $c$ on $M$ which is a solution of the differential equation

$$
\frac{D_{M}}{d u}\left(\frac{\sigma^{i}}{d u}\right)=m_{i}\left(\frac{d s}{d u}\right)^{2} \quad(i=1, \ldots, n) .
$$

When we consider the parameter $u$ as a time (2.12) means that the acceleration vector is proportional to the gradient vector $\left(m_{i}\right)$, proportion being a square of the speed. To such a curve $c$ on $M$ corresponds a curve $\varphi(c)$ on $N$ satisfying

$$
\frac{D_{N}}{d u}\left(\frac{\sigma^{i}}{d u}\right)=2 \frac{d l}{d u}-\frac{\sigma^{i}}{d u} \quad(i=1, \ldots, n) .
$$


Thus $\varphi(c)$ is a geodesic and the arc-length $t$ on it is given by

$$
t=\int e^{2 l} d u \times c o n s t
$$

In fact we have $\log (d t / d u)=2 l+$ const. in this case and we get by (2.13)

$$
\begin{aligned}
\frac{D_{N}}{d t}\left(\frac{\sigma^{i}}{d t}\right) & =\frac{d u}{d t} \frac{D_{N}}{d u}\left(\frac{\sigma^{i}}{d u} / \frac{d t}{d u}\right)=\left(\frac{d u}{d t}\right)^{2}\left[\frac{D_{N}}{d u}\left(\frac{\sigma^{i}}{d u}\right)-\frac{\sigma^{i}}{d u} \frac{d^{2} t}{d u^{2}} / \frac{d t}{d u}\right] \\
& =\left(\frac{d u}{d t}\right)^{2}\left[\frac{D_{N}}{d u}\left(\frac{\sigma^{i}}{d u}\right)-\frac{\sigma^{i}}{d u} \cdot 2 \frac{d l}{d u}\right]=0 .
\end{aligned}
$$

Thus we have got the following result.

THEOREM 3. By the mapping satisfying the relation (2.1) the curve $c$ of the solution of the differential equation (2.12) is mapped onto a geodesic on $N$, and there exists a relation (2.14) between the parameter $u$ and the arc-length $t$ of $\varphi(c)$.

The converse is also true as is shown next. We assume a mapping $\varphi$ satisfies the conclusion of the theorem 3 and denote its second tensor by $r_{j}^{i}$ and put

$$
\varepsilon_{j}^{i}=e_{j k}^{i} \sigma^{k}=\gamma_{j}^{i}-\left(\delta_{j}^{i} d l+l_{j} \sigma^{i}-m_{i} \sigma^{j}\right)
$$

Then we have

$$
\lambda_{j}^{i}=\sigma_{j}^{i}+\gamma_{j}^{i}=\sigma_{j}^{i}+\left(\delta_{j}^{i} d l+l_{j} \sigma^{i}-m_{i} \sigma^{j}\right)+\varepsilon_{j}^{i}
$$

and by a calculation analogous to (2.1) we get

$$
\frac{D_{N}}{d u}\left(\frac{\sigma^{i}}{d u}\right)=\frac{D_{M}}{d u}\left(\frac{\sigma^{i}}{d u}\right)-m_{i}\left(\frac{d s}{d u}\right)^{2}+2 \frac{d l}{d u} \frac{\sigma^{i}}{d u}+\frac{\sigma^{j}}{d u} \frac{\varepsilon_{j}^{i}}{d u} .
$$

Along a curve $c$ satisfying (2.12) we have (2.13) by assumption and so

$$
\frac{\sigma^{j}}{d u} \frac{\varepsilon_{j}^{i}}{d u}=e_{j k}^{i} \frac{\sigma^{j}}{d u} \frac{\sigma^{k}}{d u}=0
$$

along $c$. As the curve $c$ is a solution of the differential equation of the second order $(2.13)\left(\sigma^{i} / d u\right)$ can be taken as an arbitrary vector at any point. Moreover we have $e_{j k}^{i}=e_{k j}^{i}$ by (1.9) and (2.2) and we get from (2.15) $\boldsymbol{e}_{j k}^{i}=0$. Thus the converse of theorem 3 is proved.

As our mapping which satisfies (2.1) is a generalization of a projective mapping and theorem 3 holds good, we call our mapping pseudo-projective and in short a $p$-mapping hereafter. 
8. We take three Riemannian manifolds of dimension $n$ and two mappings $\psi: L \rightarrow M$ and $\varphi: M \rightarrow N$. When $\psi$ and $\varphi$ are $p$-mappings, the mapping $\varphi \circ \psi:$ $L \rightarrow N$ is not necessarily a $p$-mapping, as is shown in the next.

We use the notations in 5. Then the second tensors of $\psi$ and $\varphi$ are

$$
\begin{array}{ll}
\beta_{j}^{i}=\delta_{j}^{i} d h+h_{j} \rho^{i}-k_{i} \rho^{j} & \left(d h=h_{i} \rho^{i}\right) \\
\gamma_{j}^{i}=\delta_{j}^{i} d l+l_{j} \sigma^{i}-m_{i} \sigma^{j} & \left(d l=l_{i} \sigma^{i}\right) .
\end{array}
$$

When we put $Q=\left(q_{j}^{i}\right)$ and $Q^{-1}=\left(Q_{j}^{i}\right)$, we have for $\alpha=\left(\alpha_{j}^{i}\right)=\beta+\operatorname{Qr} Q^{-1}$

$$
\begin{aligned}
\alpha_{j}^{i}= & \beta_{j}^{i}+q_{j}^{k} \gamma_{k}^{h} Q_{h}^{i} \\
= & \delta_{j}^{i} d h+h_{j} \rho^{i}-k_{i} \rho^{j}+q_{j}^{k}\left(d l \delta_{k}^{h}+l_{k} \sigma^{h}-m_{h} \sigma^{k}\right) Q_{h}^{i} \\
= & \delta_{j}^{i} d(h+l)-\left(h_{j}+l_{j}^{\prime}\right) \rho^{i}-\left(k_{i} \rho^{j}+m_{h} q_{j}^{k} q_{n}^{k} Q_{h}^{i} \rho^{n}\right) \\
& \quad\left(d l=l_{i} \sigma^{i}=l_{i}^{\prime} \rho^{i}\right) .
\end{aligned}
$$

This is not of the form (2.1) in general.

Especially if $\varphi$ is projective $\left(m_{h}=0\right), \varphi \circ \psi$ is a $p$-mapping. Thus we get the following theorem.

TheOREM 4. If $\psi: L \rightarrow M$ is a p-mapping and $\varphi: M \rightarrow N$ is projective, then $\varphi \circ \psi: L \rightarrow N$ is a p-mapping.

The inverse of a $p$-mapping is not a $p$-mapping in general, and we can prove the following theorem.

THEOREM 5. The inverse $\varphi^{-1}$ of a p-mapping $\varphi$ is a p-mapping when and only when $\varphi$ is conformal or projective.

Proof. I.et $\varphi: M \rightarrow N$ be a $p$-mapping with its inverse $\varphi^{-1}$. In the notation we have used

$$
r=P_{\tau} P^{-1}+d P P^{-1}-\sigma .
$$

We drop $\varphi^{*}$ for the sake of convenience. We get from this

$$
-P^{-1} \gamma P=P^{-1} \sigma P+d\left(P^{-1}\right) P-\tau .
$$

In comparison with $(2.16)$ we see that $-P^{-1} \gamma P$ is the second tensor of the mapping $\varphi^{-1}$. When we put

$$
-P^{-1} \gamma P=\left(\varepsilon_{j}^{i}\right), \quad P=\left(p_{j}^{i}\right), \quad P^{-1}=\left(P_{j}^{i}\right)
$$

we get

$$
\varepsilon_{j}^{i}=-P_{j}^{k} \gamma_{k}^{h} p_{h}^{i}=-P_{j}^{k}\left(\delta_{k}^{h} d l+l_{k} \sigma^{h}-m_{h} \sigma^{k}\right) p_{h}^{i}
$$




$$
=\delta_{j}^{i} d(-l)+\left(-l_{k} P_{j}^{k}\right) \tau^{i}+m_{h} p_{h}^{j} P_{l}^{k} P_{i}^{k} \tau^{l} .
$$

As we have $-l_{k} P_{i}^{k} \tau^{i}=-l_{k} \sigma^{k}=-d l$, $\varepsilon_{j}^{i}$ is of the form (2.1) when and only when

$$
P_{l}^{k} P_{i}^{k} p_{h}^{j} m_{h}=r_{j} \delta_{i l}
$$

If there exists one $j$ such that $p_{h}^{j} m_{h} \neq 0$, we have $P_{k}^{l} P_{i}^{k}=b \delta_{l i}$ on an open set. As $\left(P_{l}^{k} P_{i}^{k}\right)$ is a positive definite matrix, $b$ is positive and $\left(b^{-\frac{1}{2}} P_{i}^{k}\right)$ is orthogonal and so is $\left(b^{\frac{1}{2}} p_{i}^{k}\right)$. Hence $\left(a_{i j}\right)=\left(p_{i}^{k} p_{j}^{k}\right)=\left(b^{-1} \delta_{i j}\right)$ and $\varphi$ is conformal. On the other hand, if $p_{h}^{j} m_{h}=0$ for all $j$ we get $m_{h}=0$ and our mapping is projective.

9. Here we treat the case $M$ is of constant curvature and $N$ is flat. Then we have $T=0$ and we get from (1.13) $\Gamma=-\sum$. As $\sum_{j}^{i}=-K \sigma^{j} \wedge \sigma^{i}$ by our assumption we get for the $p$-mapping

$$
\left(D l_{j}-l_{j} d l\right) \wedge \sigma^{i}-\left(D m_{i}-m_{i} d m\right) \wedge \sigma^{j}+l_{k} m_{k} \sigma^{j} \wedge \sigma^{i}=-K \sigma^{j} \wedge \sigma^{i}
$$

by virtue of $(2.5)$. Thus, putting

$$
D l_{j}-l_{j} d l=\left(\nabla_{k} l_{j}-l_{j} l_{k}\right) \sigma^{k}=l_{j k} \sigma^{k}, \quad D m_{i}-m_{i} d m=\left(\nabla_{h} m_{i}-m_{i} m_{h}\right) \sigma^{h}=m_{i} \sigma^{h}
$$

we get $l_{j k}=l_{k j}, m_{i h}=m_{h i}$. Hence (2.17) reduces to

$$
l_{j k} \sigma^{k} \wedge \sigma^{i}-m_{i k} \sigma^{k} \wedge \sigma^{j}+l_{k} m_{k} \sigma^{j} \wedge \sigma^{i}=-K \sigma^{j} \wedge \sigma^{i} .
$$

If $n \geqq 3$, we get $l_{j k}=0(j \neq k), m_{i h}=0 \quad(i \neq h)$,

$$
l_{j j}+m_{j j}+l_{k} m_{k}=-K \quad(\text { not summed for } i, j) .
$$

Hence we can put $l_{i i}=\lambda, m_{j j}=\mu$ (not summed for $i, j$ ), and we get

$$
l_{i j}=\lambda \delta_{i j}, \quad m_{i j}=\mu \delta_{i j}, \quad \lambda+\mu+l_{k} m_{k}=-K .
$$

If $n=2$, we get

$$
l_{11}+m_{22}=l_{22}+m_{11}=-\left(l_{1} m_{1}+l_{2} m_{2}+K\right), \quad l_{12}=m_{12},
$$

and these can not be simplified. Thus we get the following theorem.

Theorem 5. Let $M$ be a Riemannian manifold of constant curvature $K$ and $N$ is a flat Riemannian manifold, each of dimesion $n$ ( $\geqq 3)$. Then a p-mapping $M \rightarrow N$ satisfies the relations (2.18).

We give here a geometric interpretation of the second relation in (2.18)

$$
m_{i j}=\nabla_{i} m_{j}-m_{i} m_{j}=\mu \delta_{i j}
$$


THEOREM 6. If a p-mapping $\varphi$ satisfies the relation (2.19), the curves $c$ on $M$, which are mapped upon geodesics of $N$, are Riemannian circles or geodesics. Here we mèan by Riemannian circles those curves on $M$ whose delopments into the euclidean space are circles.

Proof. As a preliminary we shall show that a curve $x=x(s)$ in the euclidean space is a circle or a straight line when and only when $d^{3} x / d s^{3}$ is collinear with $d x / d s$, where $s$ means an arclength along the curve. Necessity is evident from Frenet-Serret's formula. We prove the sufficiency. By assumption we have $x^{\prime \prime \prime}=h x^{\prime}$ (' means derivation with respect to $s$ ). Since $\left(x^{\prime}, x^{\prime \prime}, x^{\prime \prime \prime}\right)=0$ the curve $c$ is a plane curve. We have on the other hand $\left(x^{\prime}, x^{\prime}\right)=1,\left(x^{\prime}, x^{\prime \prime}\right)=0$, $\left(x^{\prime \prime}, x^{\prime \prime}\right)+\left(x^{\prime}, x^{\prime \prime \prime}\right)=0$ and hence $h=-k^{2}$ ( $k$ being a curvature). When $k \neq 0$, $k^{-1} x$ is a unit principal normal and by Frenet's formula for a plane curve we have $\left(k^{-1} x^{\prime \prime}\right)^{\prime}=-k x^{\prime}$, and taking $x^{\prime \prime \prime}=-k^{2} x^{\prime}$ into consideration we get $k^{\prime}=0$, which was to be proved in preliminary (cf. [6]).

The condition for $d^{3} x / d s^{3}$ to be collinear with $d x / d s$ is equivalent to the property

$$
\frac{d^{3} x}{d s^{3}}\left(\frac{d x}{d u}\right)^{2}-3 \frac{d^{2} x}{d s^{2}} \frac{d s}{d u} \frac{d^{2} s}{d u^{2}} \text { is collinear with } \frac{d x}{d s}
$$

In fact we have

$$
\frac{d^{3} x}{d s^{3}}=\left(\frac{d s}{d u}\right)^{-5}\left[\frac{d^{3} x}{d u^{3}}\left(\frac{d s}{d u}\right)^{2}-3 \frac{d^{2} x}{d u^{2}} \frac{d s}{d u} \frac{d^{2} s}{d u^{2}}+\frac{d x}{d u}\left\{-\frac{d^{3} s}{d u^{3}} \frac{d s}{d u}+3\left(\frac{d^{2} s}{d u^{2}}\right)^{2}\right\}\right]
$$

On a Riemannian manifold the condition for a curve to be a Riemannian circle or a geodecic can be given by replacing differentiatiation by a covariant one in (2.20). For a curve in question we have by (2.12)

$$
\frac{D}{d u}\left(\frac{\sigma^{i}}{d u}\right)=m_{i}\left(\frac{d s}{d u}\right)^{2}
$$

where $D$ means a covariant differentiation on $M$. We put

$$
Q^{i}=\frac{D^{2}}{d u^{2}}\left(\frac{\sigma^{i}}{d u}\right)\left(\frac{d s}{d u}\right)^{2}-3 \frac{D}{d u}\left(\frac{\sigma^{i}}{d u}\right) \frac{d s}{d u} \frac{d^{2} s}{d u^{2}}
$$

and we get for the curve $c$

$$
Q^{i}=\frac{D m_{i}}{d u}\left(\frac{d s}{d u}\right)^{4}+2 m_{i}\left(\frac{d s}{d u}\right)^{3} \frac{d^{2} s}{d u^{2}}-3 m_{i}\left(\frac{d s}{d u}\right)^{3} \frac{d^{2} s}{d u^{2}}
$$




$$
=\left[\frac{D m_{i}}{d u}\left(\frac{d s}{d u}\right)^{2}-m_{i} \frac{d s}{d u} \frac{d^{2} s}{d u^{2}}\right]\left(\frac{d s}{d u}\right)^{2}
$$

Here we have

$$
\begin{aligned}
\frac{d s}{d u} \frac{d^{2} s}{d u^{2}} & =\frac{1}{2} d u\left(\frac{d s}{d u}\right)^{2}=\frac{1}{2} \frac{D}{d u}\left(\frac{\sigma^{j}}{d u} \frac{\sigma^{j}}{d u}\right)=\frac{\sigma^{j}}{d u} \frac{D}{d u}\left(\frac{\sigma^{j}}{d u}\right) \\
& =\frac{\sigma^{j}}{d u} m_{j}\left(\frac{d s}{d u}\right)^{2}=\frac{d m}{d u}\left(\frac{d s}{d u}\right)^{2}
\end{aligned}
$$

and so

$$
Q^{i}=\left(-\frac{D m_{i}}{d u}-m_{i} \frac{d m}{d u}\right)\left(\frac{d s}{d u}\right)^{4}=m_{i j} \frac{\sigma^{j}}{d u}\left(\frac{d s}{d u}\right)^{4}
$$

As $m_{i j}=\ell \delta_{i j}$ by the assumption, $Q^{i}$ in collinear with $\sigma^{i} / d s$ and our theorem is proved.

As a corollary we get the following.

THEOREM 7. If $\varphi$ is a p-mapping of a Riemannian manifold $M$ of constant curvature onto a flat Riemannian manifold $N$, each of dimension $n(\geqq 3)$, the inverse image $\varphi^{-1}(c)$ of any geodesic $c$ on $N$ is a Riemannian circle or a geodesic.

10. All the $p$-mappings from a flat $M$ to a flat $N$ can be obtained as follows when the dimension $n$ is greater than 2 . We take as local coordinates rectangular ones $x^{1}, \ldots, x^{n}$. Then we have by virtue of (2.18)

$$
l_{i k}=\frac{\partial^{2} l}{\partial x^{i} \partial x^{k}}-\frac{\partial l}{\partial x^{i}} \frac{\partial l}{\partial x^{k}}=0(i \neq k), \quad m_{j h}=\frac{\partial^{2} m}{\partial x^{j} \partial x^{h}}-\frac{\partial m}{\partial x^{j}} \frac{\partial m}{\partial x^{h}}=0(j \neq h) .
$$

Hence we get

$$
e^{-l}=\sum_{k} A_{k}\left(x^{k}\right), \quad e^{-m}=\sum_{k} B_{k}\left(x^{k}\right)
$$

As

$$
l_{i i}=\frac{\partial^{2} l}{\left(\partial x^{i}\right)^{2}}-\left(\frac{\partial l}{\partial x^{i}}\right)^{2}=\lambda, \quad m_{j j}=\frac{\partial^{3} m}{\left(\partial x^{j}\right)^{2}}-\left(\frac{\partial m}{\partial x^{j}}\right)^{2}=\mu
$$

are independent with $i$ and $j$ (not summed,) we have

$$
A_{k}^{\prime \prime}=2 a \text { (const.), } \quad B_{k}^{\prime \prime}=2 b \text { (const.). }
$$

Hence

$$
\begin{gathered}
e^{-l}=a x^{k} x^{k}+2 c_{k} x^{k}+f, \quad e^{-m}=b x^{k} x^{k}+2 d_{k} x^{k}+g \\
\left(a, b, c_{k}, d_{k}, f, g \text { constants }\right)
\end{gathered}
$$

Moreover by the relation $\lambda+\mu+l_{k} m_{k}=0$ in $(2.18)$ we get 


$$
a g+b f=2 c_{k} d_{k} .
$$

Conversely we assume that $l$ and $m$ are as in (2.21) accompanied by the relation $(2.22)$ and put

$$
\gamma_{j}^{i}=\delta_{j}^{i} d l+l_{j} d x^{i}-m_{i} d x^{j}, \quad d l=l_{i} d x^{i}, \quad d m=m_{i} d x^{i} .
$$

We solve the differential equation

$$
d a_{i j}=a_{i k} \gamma_{j}^{k}+a_{k j} \gamma_{i}^{k}
$$

with unknown $a_{i j}$. It is completely integrable because the condition for (2.24) to be completely integrable is by (1.27)

$$
a_{i k} \Gamma_{j}^{k}+a_{k i} \Gamma_{i}^{k}=0
$$

in our case and it is satisfied by $\Gamma_{i}^{j}=0$, which is evident by our construction of $l$ and $m$.

Thus the mapping which maps $M: d s^{2}=d x^{i} d x^{i}$ onto $N: d t^{2}=a_{i j} d x^{i} d x^{j}$ in such a way that the corresponding points have the same coordinates, is a $p$ mapping with $\Gamma_{j}^{i}=0$ and the curvature tensor $T$ of $d t^{2}$ vanishes by (1.13). Hence our mapping is a $p$-mapping of a flat $M$ onto a flat $N$. We will give examples of $p$-mappings which are more interesting in the next section.

\section{Examples of pseudo-projective mappings}

11. As a preliminary we consider an $n$-dimensional Riemannian metric $d u^{2}$ such that

$$
d u^{2}=p\left(x^{1}\right)^{2}\left(d x^{1}\right)^{2}+q\left(x^{1}\right)^{2} d v^{2},
$$

where $d v^{2}$ is an $n-1$ dimensional Riemannian metric independent with $x^{1}$. We take orthogonal coframe and put

$$
d v^{2}=\pi^{\alpha} \pi^{x} \quad(\alpha=2, \ldots, n)
$$

and its forms of Riemannian connection $\left(\pi_{\beta}^{\alpha}\right)$ are determined by

$$
d \pi^{\alpha}=\pi^{\beta} \wedge \pi_{\beta}^{\alpha}, \quad \pi_{\beta}^{\alpha}=-\pi_{\alpha}^{\beta} \quad(\alpha, \beta=2, \ldots, n) .
$$

We put

$$
\omega^{1}=p d x^{1}, \quad \omega^{\alpha}=q \pi^{\alpha}, \quad d q / d x^{1}=q^{\prime} \quad(\alpha=2, \ldots, n) .
$$

Then we get

$$
d u^{2}=\omega^{i} \omega^{i}
$$


and its forms of Riemannian connection $\left(\omega_{j}^{i}\right)$ are given by

$$
\omega_{1}^{1}=0, \quad \omega_{1}^{\alpha}=p^{-1} q^{\prime} \pi^{\alpha}=-\omega_{\alpha}^{1}, \quad \omega_{\beta}^{\alpha}=\pi_{\beta}^{\alpha} .
$$

In fact we have then

$$
d \omega^{i}=\omega^{j} \wedge \omega_{j}^{i}, \quad \omega_{j}^{i}=-\omega_{i}^{j} \quad(i, j=1, \ldots, n)
$$

by virtue of (3.3) (3.5).

Hereafter in this section we use the indices $\alpha, \beta, \ldots$ and $i, j, \ldots$ as indicated above.

12. We take two $n$-dimensional Riemannian manifolds $M$ and $N$ whose merics are given locally by

$$
\begin{aligned}
& M: d s^{2}=\left(d x^{1}\right)^{2}+c^{2} d v^{2} \\
& N: d t^{2}=a^{2}\left(d x^{1}\right)^{2}+b^{2} c^{2} d v^{2}
\end{aligned}
$$

with $a=a\left(x^{1}\right), b=b\left(x^{1}\right), c=c\left(x^{1}\right)$ all positive and $d v^{2}$ as in (3.2). We put

$$
\sigma^{1}=d x^{1}, \quad \sigma^{\alpha}=c \pi^{\alpha}
$$

and take these as coframes on $M$. Then we have

$$
d s^{2}=\sigma^{i} \sigma^{i}
$$

and its forms of Riemannian connection are by (3.6)

$$
\sigma_{1}^{\alpha}=c^{\prime} \pi^{\alpha}, \quad \sigma_{3}^{\alpha}=\pi_{\beta}^{\alpha} \quad\left(c^{\prime}=d c / d x^{1}\right) .
$$

and so

$$
\sigma=\left(\begin{array}{cc}
0 & c^{\prime} \pi^{\alpha} \\
-c^{\prime} \pi^{\beta} & \pi_{\beta}^{\alpha}
\end{array}\right) .
$$

Next we put

$$
\tau^{1}=a d x^{1}, \quad \tau^{\alpha}=b c \pi^{\alpha}
$$

and take these as a coframe on $N$. Then we have

$$
d t^{2}=\tau^{i} \tau^{i}
$$

and its forms of Riemannian connection are by (3.6)

$$
\tau_{1}^{\alpha}=a^{-1}(b c)^{\prime} \pi^{\alpha}, \quad \tau_{\beta}^{\alpha}=\pi_{\beta}^{\alpha}
$$

and so

$$
\tau=\left(\begin{array}{cc}
0 & a^{-1}(b c)^{\prime} \pi^{\alpha} \\
-a^{-1}(b c)^{\prime} \pi^{\beta} & \pi_{\beta}^{\alpha}
\end{array}\right) .
$$


We denote $d v^{2}$ as $d v^{2}=g_{\alpha \beta} d x^{\alpha} d x^{\beta}$ in local coordinates and consider a mapping $\varphi: M \rightarrow N$ which maps a point $\left(x^{1}, \ldots, x^{n}\right)$ of $M$ onto a point $\left(x^{1}, \ldots, x^{n}\right)$ of $N$. Then the matrix $P=\left(p_{j}^{i}\right)$ defined by $\varphi^{*} \tau^{i}=p_{j}^{i} \sigma^{j}$ in 1 is

$$
P=\left(\begin{array}{cc}
a & 0 \\
0 & b E_{n-1}
\end{array}\right)
$$

where $E_{n-1}$ is a unit matrix. Hence we get

$$
\lambda=P_{\tau} P^{-1}+d P P^{-1}=\left(\begin{array}{cl}
a^{-1} d a & b^{-1}(b c)^{\prime} \pi^{\alpha} \\
-a^{-2} b(b c)^{\prime} \pi^{\beta} & b^{-1} d b \cdot \delta_{\beta}^{\alpha}+\pi_{\beta}^{\alpha}
\end{array}\right) .
$$

By (1.8) the second tensor is $\gamma=\left(\gamma_{j}^{i}\right)=\lambda-\sigma$ and by (3.12) (3.15)

$$
\begin{aligned}
& \gamma_{1}^{1}=a^{-1} d a=a^{-1} a^{\prime} d x^{1}, \quad \gamma_{\alpha}^{\alpha}=b^{-1} d b=b^{-1} b^{\prime} d x^{1} \quad(\text { not summed for } \alpha \text { ) } \\
& \gamma_{1}^{\alpha}=b^{-1} b^{\prime} c \pi^{\alpha}, \quad \gamma_{\alpha}^{1}=\left(c^{\prime}-a^{-2} b(b c)^{\prime}\right) \pi^{\alpha}, \quad \gamma_{3}^{\alpha}=0 \quad(\alpha \neq \beta)
\end{aligned}
$$

We seek for conditions for $\varphi$ to be a $p$-mapping. This is by definition

$$
r_{j}^{i}=\delta_{j}^{i} d l+l_{j} \sigma^{i}-m_{i} \sigma^{j}
$$

We assume $\varphi$ is a $p$-mapping, and we have

$$
\left.r_{1}^{1}=d l+\left(l_{1}-m_{1}\right) d x^{1}, \quad \gamma_{\alpha}^{\alpha}=d l+\left(l_{\alpha}-m_{\alpha}\right) \sigma^{\alpha} \text { (not summed for } \alpha\right)
$$

By taking their difference and taking (3.16) into consideration we get

$$
\left(l_{1}-m_{1}\right) d x^{1}-\left(l_{\alpha}-m_{\alpha}\right) \sigma^{\alpha}=\left(a^{-1} a^{\prime}-b^{-1} b^{\prime}\right) d x^{1}
$$

and so

$$
l_{\alpha}=m_{\alpha} .
$$

Hence by (3.18) (3.16)

$$
d l=b^{-1} b^{\prime} d x^{1}
$$

Then we have $l_{\alpha}=0$ and so $m_{\alpha}=0$. Hence we get by (3.18) (3.16)

$$
d m=m_{1} d x^{1}=d l+l_{1} d x^{1}-\gamma_{1}^{1}=2 d l-a^{-1} d a
$$

and then by (3.19)

$$
d m=2 b^{-1} d b-a^{-1} d a
$$

By (3.19) and (3.20) we get

$$
l=\log b+\text { const., } \quad m=\log \left(a^{-2} b\right)+\text { const. }
$$

Next we have by (3.16) 


$$
r_{\alpha}^{1}=\left(c^{\prime}-a^{-2} b(b c)^{\prime}\right) \pi^{\alpha} .
$$

On the other hand by (3.20)

$$
l_{\alpha} \sigma^{1}-m_{1} \sigma^{\alpha}=-m_{1} \sigma^{\alpha}=-m^{\prime} \sigma^{\alpha}=\left(a^{-1} a^{\prime}-2 b^{-1} b^{\prime}\right) c \pi^{\alpha} .
$$

As we have by (3.17)

$$
\gamma_{\alpha}^{1}=l_{\alpha} \sigma^{1}-m_{1} \sigma^{\alpha}
$$

we get

$$
c^{\prime}-a^{-2} b(b c)^{\prime}=\left(a^{-1} a^{\prime}-2 b^{-1} b^{\prime}\right) c .
$$

This is equivalent to

$$
\left(a^{2}-b^{2}\right) c^{-1} c^{\prime}=-2\left(a^{2}-b^{2}\right) b^{-1} b^{\prime}+\left(a a^{\prime}-b b^{\prime}\right)
$$

and is also to

$$
a=b \quad \text { or } \quad c^{2}=b^{-4}\left|a^{2}-b^{2}\right| \times \text { const. }
$$

Conversely we assume (3.24). We take $l$ and $m$ given by (3.21). Then we have (3.18) and (3.22), and by (3.16) (3.21) (3.9)

$$
\gamma_{1}^{\alpha}=b^{-1} b^{\prime} c \pi^{\alpha}=l_{1} \sigma^{\alpha}
$$

and so

$$
\gamma_{1}^{\alpha}=l_{1} \sigma^{\alpha}-m_{\alpha} \sigma^{1}
$$

By (3.16) and $l_{\alpha}=0, m_{\alpha}=0$ we have

$$
\gamma_{\beta}^{\alpha}=l_{\beta} \sigma^{\alpha}-m_{\alpha} \sigma^{\beta} \quad(\alpha \neq \beta) .
$$

Thus (3.17) has been verified, and we have got the following theorem.

THEOREM 8. We assume that the metrics of two Riemannian manifolds $M$ and $N$ are given by (3.7) and (3.8). In order that a mapping which maps a point of $M$ to the point of $N$ with the same coordinates is a p-mapping, it is necessary and sufficient that the functions $a, b, c$ satisfy (3.24).

13. We will show here that in the euclidean space $E_{n+1}$ a mapping of a hypersphere onto a hyperplane, which is induced by a projection through a fixed point, is a $p$-mapping. In the first we take a unit sphere $S$ and a hyperplane $L$ which touches $S$ at a point $O$. We choose a fixed point $A$ on the diameter of $S$ through $O$ and project from $A$ a point $P$ on $S$ onto a point $Q$ on $L$. We denote by $x^{1}$ an angle between two radii of $S$ whose ends are points $O$ and $P$, and denote the lengths of segments $O A$ and $O Q$ by $k$ and $r$ respectively. 
Now we take rectangular axis with the origin at $O$, and $A$ on the $n+1$ -th axis. We represent the rectangular coordinates of a point $Q$ on $L$ by $\left(r l_{1}\right.$, $\left.\ldots, r l_{n}, 0\right)$. Then $\left(l_{1}, \ldots, l_{n}, 0\right)$ are components of a unit vector at $O$ and the metric on the hyperplane $L$ induced from the eucliciean metric in $E_{n+1}$ is

$$
d t^{2}=d r^{2}+r^{2} d v^{2}
$$

where $d v^{2}$ is a metric on an $n-1$ dimensional unit sphere on $L$. The coordinates of the point $P$ on $S$ which is projected on $Q$ by a straight line through $A$ is $\left(l_{1} \sin x^{1}, \ldots, l_{n} \sin x^{1}, 1-\cos x^{1}\right)$. Hence the induced metric on $S$ is

$$
d s^{2}=\left(d x^{1}\right)^{2}+\left(\sin x^{1}\right)^{2} d v^{2}
$$

and the relation between $r$ and $x$ is given by

$$
r=\frac{k \sin x^{1}}{k-1+\cos x^{1}}
$$

as simple calculation shows. We put

$$
a=\frac{k\left(1+(k-1) \cos x^{1}\right)}{\left(k-1+\cos x^{1}\right)^{2}}, \quad b=\frac{k}{k-1+\cos x^{1}}, \quad c=\sin x^{1} .
$$

Then (3.28) and (3.27) can be written as

$$
d s^{2}=\left(d x^{1}\right)^{2}+c^{2} d v^{2}, \quad d t^{2}=a^{2}\left(d x^{1}\right)^{2}+b^{2} c^{2} d v^{2} .
$$

In case $k=2$ we have $a=b$ and in case $k \neq 2$ we have $c^{2}=b^{-4}\left(b^{2}-a^{2}\right)$. Hence our projection is a $p$-mapping by theorem 8 . We can assert a more general theorem as follows.

THEOREM 9. In the euclidean space we project a hypersphere $S$ on a hyperplane $L$ by a straight line through a fixed point. This mapping is p-mapping.

Proof. We take a hyperplane $L_{1}$ which is tangent to $S$ at the point where the diameter through the fixed point $A$ intersects $S$. The mapping $\varphi$ in the theorem can be decomposed as $\varphi=\psi \circ \varphi_{1}$, where $\varphi_{1}$ is a projection of $S$ onto $L_{1}$ with the center at $A$ and $\psi$ is a projection of $L_{1}$ onto $L$ with the same center. As $\varphi_{1}$ is a $p$-mapping and $\psi$ is projective, $\dot{\psi} \circ \varphi_{1}$ is a $p$-mapping by theorem 4 .

14. Finally we will give an example of a $p$-mapping in the euclidean space. We take two euclidean spaces $E_{n}$ and $E_{n}^{\prime}$ of dimension $n$. In $E_{n}$ the euclidean metric is given by

$$
d s^{2}=d r^{2}+r^{2} d v^{2}
$$


where $r$ is a distance from a fixed point (origin) to an arbitrary point and $d v^{2}$ is the induced metric on a unit hypersphere with the center at the origin. If the metric of $E_{n}^{\prime}$ is given by

$$
d t^{2}=a^{2} d r^{2}+(b r)^{2} d v^{2} \quad(a=a(r), b=b(r))
$$

with

$$
r^{2}=b^{-4}\left|a^{2}-b^{2}\right| \times \text { const., }
$$

then the mapping which maps the point on $E_{n}$ onto the point on $E_{n}^{\prime}$ with the same coordinates, is a $p$-mapping by theorem 8 . Now the metric (3.31) is flat if

$$
d(b r)=a d r
$$

by the same reason as (3.30). We will find $a$ and $b$ satisfying (3.32) and (3.33). We put $b r=w$ and denote the constant in (3.32) by $k$. Then we get

$$
a=\frac{d w}{d r}, \quad\left(\frac{d w}{d r}\right)^{2}-\left(\frac{w}{r}\right)^{2}= \pm k \frac{w^{4}}{r^{2}} .
$$

By solving the second equation we get

$$
w=\frac{r}{k_{1} r^{2}+k_{2}} \quad\left(k_{1}, k_{2} \text { constants }\right)
$$

Now (3.31) can be written as

$$
d t^{2}=d w^{2}+w^{2} d v^{2}
$$

When we represent $d v^{2}$ as $d v^{2}=g_{i j} d x^{i} d x^{j}(i, j=1, \ldots, n-1)$, the mapping which maps the point with polar coordinates $\left(r, z^{1}, \ldots, z^{n-1}\right)$ of $E_{n}$ to the point with coordinates $\left(w, z^{1}, \ldots, z^{n-1}\right)$ on $E_{n}^{\prime}$ is a $p$-mapping. When we superpose $E_{n}$ and $E_{n}^{\prime}$, in such a way that the two points with the same polar coordinates coinside, we get the following theorem.

THEOREM 10. In the euclidean space we take a fixed point $O$ and maps any point $P$ onto a point $Q$ on the straight line $O P$ in such a way that between $O P=r$ and $O Q=w$ the relation

$$
w=\frac{r}{k_{1} r^{2}+k_{2}} \quad\left(k_{1}, k_{2} \text { constants }\right)
$$

holds good. This mapping is a p-mapping.

In case $k_{1}=0$ our mapping is a similar mapping and in case $k_{2}=0$ an 
inversion. In rectagular coordinates the mapping $\left(x^{1}, \ldots, x^{n}\right) \rightarrow\left(X^{1}, \ldots, X^{n}\right)$ in theorem 10 is represented as

$$
X^{i}=\frac{x^{i}}{k_{1} r^{2}+k_{2}} \quad\left(r^{2}=x^{i} x^{i}\right)
$$

Especially if $k_{1}=-1$ and $k_{2}=1$ we get

$$
X^{i}=\frac{x^{i}}{1-r^{2}} \quad\left(r^{2}=x^{i} x^{i}\right)
$$

and this mapping maps the interior of the unit sphere $S: x^{i} x^{i}=1$ onto the whole euclidean space. It is a hypersphere or a hyperplane

$$
A_{i} x^{i}=B\left(1-x^{i} x^{i}\right) \quad\left(A_{i}, B \text { const. }\right)
$$

which passes through the intersection of the unit hypersphere $S$ and a hyperplane $A_{i} x^{i}=0$ that is mapped upon a hyperplane. Hence it can be easily be shown that it is an arc of a circle joining two end points of a diameter of $S$ that is mapped onto a straight line by our mapping. Thus we get a representation of the whole euclidean space in a unit hypersphere $x^{i} x^{i}=1$ whose euclidean metric is

$$
d s^{2}=d X^{i} d X^{i}=\left(1-r^{2}\right)^{-2} d x^{i} d x^{i}+4\left(1-r^{2}\right)^{-4} r^{2}(d r)^{2} \quad\left(r^{2}=x^{i} x^{i}\right)
$$

where the straight lines are replaced by arcs of circles joining two end points of diameters of the fundamental unit hypersphere.

\section{REFERENCES}

[1] Adati, T., On subprojective spaces I-VI, Tôhoku Math. J. 3 (1951), pp. 159-173, 330342, 343-358; Tensor I (1951), pp. 105-115, 116-129, 130-136.

[2] Chern, S. S., Topics in differential geometry. Mimeographed note. The institute of advanced study, Princeton (1951).

[3] Hano, J. On affine transformations of a Riemannian manifold. Nagoya Math. J. 9 (1955), pp. 99-109.

[4] Kobayashi, S., A theorem on the affine transformation group of a Riemannian manifold. Nagoya Math. J. 9 (1955), pp. 39-42.

[5] Kurita, M., On conformal Riemann spaces. J. Math. Soc. Japan 7 (1955), pp. 13-31.

[6] Kurita, M., Geodesic correspondence of Riemann spaces. J. Math. Soc. Japan (1956), pp. 22-39.

[7] Yano, K., Concircular geometry, I Concircular transformations. Proc. Imp. Acad. Japan (1940), pp. 195-200. 
[8] Yano, K. Subprojective transformatious, subprojective spaces and subprojective collineations. Proc. Imp. Acad. Japan (1944), pp. 701-705.

[9] Yano, K., On harmonic and Killing vector fields. Annals of Math. 55 (1952), pp. 38-45.

Mathematical Institute

Nagoya University 